\title{
INVERTIBILITY IN NEST ALGEBRAS
}

\author{
AVRAHAM FEINTUCH AND ALAN LAMBERT
}

\begin{abstract}
Let $₹$ denote a complete nest of subspaces of a complex Hilbert space $\mathcal{H}$, and let $\mathcal{C}$ denote the nest algebra defined by $\mathcal{F}$. Let $\mathcal{K}$ denote the ideal of compact operators on $\mathcal{H}$. If $\mathcal{F}$ has no infinite-dimensional gaps then $T \in C$ is invertible in $C$ if and only if it is invertible in $C+K$. An example is given of a nest with an infinite gap for which there exists an operator in $C$ which is invertible in $C+K$ but not in $C$.
\end{abstract}

1. Introduction. The idea of looking at a chain of invariant subspaces of an operator and relating it to the structure of the operator and its spectrum, is basic to finite-dimensional linear algebra. The first attempt to generalize such an approach for nonselfadjoint operators in Hilbert space seems to have been in a classical paper of M. Lifshitz [6]. This was later pursued by a number of mathematicians in the Soviet Union and is, for the most part, summarized in [5].

Independently, J. Ringrose [7] defined the notion of a nest algebra, an algebra of operators which leaves a chain of subspaces invariant, and studied the basic properties of such algebras. We are concerned with the question of identifying the invertible elements of a nest algebra $C$. While it is always of interest to identify the invertible elements of a Banach algebra, in this case the problem has significance in the stability theory of input-output systems. The interested reader is referred to $[\mathbf{3}, \mathbf{4}]$.

It was shown in [2] that if $\mathcal{K}$ represents the ideal of compact operators on $\mathcal{H}$ and $C$ is a nest algebra, then $C+K$ is a Banach algebra.

Here we show, that if a complete nest $\mathcal{F}$ has only finite-dimensional gaps and

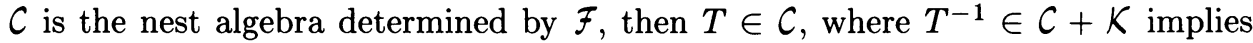
$T^{-1} \in C$. This corrects the proof of and extends a previously announced result in this direction $[8]$.

2. Preliminaries. Let $\sharp$ be a separable Hilbert space. A family of subspaces $\mathcal{F}$ of $\sharp$ will be called a nest if it is totally ordered by inclusion. $\mathcal{F}$ is complete if:

(i) $\{0\}, \forall \in \mathcal{F}$;

(ii) given any subnest $\mathcal{F}_{0} \subset \mathcal{F}$, the subspaces $\bigcap\left\{L: L \in \mathcal{F}_{0}\right\}$ and $\bigvee\left\{L: L \in \mathcal{F}_{0}\right\}$ are both members of $\mathcal{F}$.

Given a complete nest $\mathcal{F}$ and a nonzero subspace $M$ in $\mathcal{F}$, we define the predecessor $M_{-}$of $M$ by

$$
M_{-}=\bigvee\{L: L \in \mathcal{F}, L \subset M, L \neq M\}
$$

Received by the editors January 19, 1983 and, in revised form, August 25, 1983.

1980 Mathematics Subject Classification. Primary 47C05, 47A15, 47B05. 
If $C$ is the family of operators defined by $C=\{A \in B(\not H): A L \subseteq L$, for all $L \in \mathcal{F}\}$, it is easily seen that $C$ is a weakly closed algebra containing the identity. $C$ is called the nest algebra associated with $\mathcal{F}$.

Suppose $K$ is the ideal of compact operators in $B(\mathcal{H})$. The following is proved in $[\mathbf{2}]$.

THEOREM A. $C+K$ is a norm closed algebra. The natural isomorphism of $\mathrm{C} / \mathrm{C} \cap \mathrm{K}$ and $\mathrm{C}+\mathrm{K} / \mathrm{C}$ is a Banach algebra isomorphism.

3. The main result. Suppose $T \in C$ is an invertible operator on $\forall$ whose inverse is in $C+K$. Then $T^{-1}=A+K$ with $A \in C, K \in \mathcal{K}$. Thus $I=T T^{-1}=$ $T A+T K$, and $T K=I-T A$ is a compact operator in $C$. If $₹$ is a continuous nest $\left(M_{-}=M\right.$ for all $\left.M \in \mathcal{F}\right)$, it follows from a result of Gohberg-Krein [5] or Ringrose [7] that $T K$ is quasinilpotent. Thus $(I-T K)$ is invertible and $(I-T K)^{-1}$ is in fact a power series in $T K$. It follows that $(I-T K)^{-1}$ and, therefore $T^{-1}=A(I-T K)^{-1}$, is in $C$.

This argument does not work if $\mathcal{F}$ is not continuous, since $I-T K$ may not be invertible. We will show that if for all $M \in \mathcal{F}, \operatorname{dim}\left(M \ominus \mathcal{M}_{-}\right)<\infty$, then the same property holds. We begin with a preliminary lemma.

LEMMA 1. Suppose $T$ is an operator on $\sharp$ such that the restriction of $T$ to an invariant subspace of finite codimension is invertible. If $T$ has trivial kernel, then $T$ is invertible on $H$.

ProOF. Suppose $\mathcal{H}=\mathcal{M} \oplus \mathcal{M}^{\perp}$ and $T$ has the matrix representation

$$
T=\left(\begin{array}{cc}
T_{1} & T_{2} \\
0 & T_{3}
\end{array}\right)
$$

with respect to this decomposition. Then, it suffices to show that $T_{3}=$ $\left(I-P_{\mathcal{M}}\right) T\left(I-P_{\mathcal{M}}\right)$ is invertible on $\mathcal{M}^{\perp}$. Since $\mathcal{M}^{\perp}$ is finite dimensional we show $T_{3}$ has trivial kernel. If $x \in \mathcal{M}^{\perp}$ such that $T_{3} x=0$, this implies $T x \in \mathcal{M}$. But then $T x=T_{1} y=T y$ for some $y \in \mathcal{M}$. But this is impossible since $T$ has trivial kernel and $x \in \mathcal{M}^{\perp}$.

THEOREM 2. Let $\mathcal{F}$ be a complete nest on $\sharp$ with no infinite-dimensional gaps. If $C$ is the nest algebra determined by $₹$ then $T \in C$ and $T^{-1} \in C+\mathcal{K}$ implies $T^{-1} \in C$.

Proof. Suppose $\mathcal{M} \in \mathcal{F}$. Since, as above, $T A=I-T K$ implies $T K \in \mathcal{C}$ and $T K$ is compact, it is enough to consider the case where $P_{\mathcal{M}}(I-T K) \mid \mathcal{M}$ is not invertible. For otherwise, $(I-T K) \mathcal{M}=\mathcal{M}$ implies $\mathcal{M}=T A \mathcal{M}$ and therefore $\mathcal{M} \supset A \mathcal{M}=T^{-1} \mathcal{M}$.

If $P_{\mathcal{M}}(I-T K) \mid \mathcal{M}$ is not invertible, then, since $T K$ is compact, there exist at most finitely many $\left\{L_{i}\right\}_{i=1}^{n} \in \mathcal{F}$ with $L_{i} \neq L_{i-}$ such that $L_{i} \ominus L_{i-}$ contains a vector $x_{i}$ with $(I-T K) x_{i} \in L_{i-}$. Assume $L_{1} \subset L_{2} \subset \cdots \subset L_{n} \subset \mathcal{M}$.

If $\left(L_{1}\right)_{-}=\{0\}$, then $L_{1}$ is a finite-dimensional subspace invariant under $T$ and thus, by a simple dimension argument, $T L_{1}=L_{1}$.

If $\left(L_{1}\right)_{-} \neq\{0\}$, then by the assumption on $\left\{L_{i}\right\}_{i=1}^{n}, P_{\left(L_{1}\right)_{-}}(I-T K) \mid\left(L_{1}\right)_{-}$is invertible. By the argument of the first paragraph of the proof, we obtain $T\left(L_{1}\right)_{-}=$ $\left(L_{1}\right)_{\ldots}$. Applying Lemma 1 to $T$ gives $T L_{1}=L_{1}$. 
Now consider $L_{2}$, which we write as $L_{2}=\left(L_{2} \ominus L_{1}\right) \oplus L_{1}$. Let $\hat{S}$ denote the compression of an operator $S \in C$ to $L_{1}^{\perp}$. Since $T L_{1}=L_{1}$, to show $T L_{2}=L_{2}$ it suffices to show that $\hat{T}\left(L_{2} \ominus L_{1}\right)=L_{2} \ominus L_{1}$.

If $L_{1}=\left(L_{2}\right)_{-}$, then $L_{2} \ominus L_{1}$ is finite dimensional. Noting that $T$ is invertible and $T L_{1}=L_{1}$ implies $\hat{T}$ is invertible, and that $\hat{T}\left(L_{2} \ominus L_{1}\right) \subset\left(L_{2} \ominus L_{1}\right)$, it follows that $\hat{T}\left(L_{2} \ominus L_{1}\right)=L_{2} \ominus L_{1}$.

If $L_{1} \neq\left(L_{2}\right)_{-}$, then $\hat{P}_{\left(L_{2}\right)_{-}}(\hat{I}-\hat{T} \hat{K}) \mid\left[\left(L_{2}\right)_{-} \ominus L_{1}\right]$ is invertible. Thus by the argument given in the first paragraph of the proof, $\hat{T}\left[\left(L_{2}\right)_{-} \ominus L_{1}\right]=\left[\left(L_{2}\right)_{-} \ominus L_{1}\right]$. By Lemma 1, applied to $\hat{T}$ we obtain $\hat{T}\left(L_{2} \ominus L_{1}\right)=L_{2} \ominus L_{1}$ and thus $T L_{2}=L_{2}$.

Since $\mathcal{M}=\left(\mathcal{M} \ominus L_{n}\right) \oplus\left(L_{n} \ominus L_{n-1}\right) \oplus \cdots \oplus\left(L_{2} \ominus L_{1}\right) \oplus L_{1}$, the above argument applied $n$ times gives $T \mathcal{M}=\mathcal{M}$ and completes the proof.

What happens when $₹$ has infinite-dimensional gaps? Before we answer this we give a result to show that this question for compact operators is equivalent to the question for finite rank operators. This may be of independent interest.

THEOREM 3. Suppose that for $T \in C, T^{-1}=A+F, F$ finite rank, implies $F \in C$. Then $T \in C$ and $T^{-1}=A+K, K$ compact, implies $K \in \mathcal{C}$.

Proof. Suppose $T \in C$ with $T^{-1}=A+K$. We show that for every $\varepsilon>0$, where $\varepsilon<1 /\|T\|$, there exists an invertible operator $B_{\varepsilon}^{-1}=L+F$ with $L \in C, F$ finite rank, $B_{\varepsilon} \in C$ and $\left\|B_{\varepsilon}^{-1}-T^{-1}\right\|<\varepsilon$. Since, by hypothesis, $B_{\varepsilon}^{-1} \in C$, this will imply $T^{-1} \in C$.

If $\left\|B_{\varepsilon}^{-1}-T^{-1}\right\|<1 /\|T\|$, it follows that $B_{\varepsilon}=T \sum_{i=0}^{\infty}\left(I-B_{\varepsilon}^{-1} T\right)^{i}$. Thus if $B_{\varepsilon}^{-1} T \in \mathcal{C}$ so is $B_{\varepsilon}$. As we have seen, $T^{-1}=A+K$ implies $K T \in C$. Thus [1] there exists a sequence $\left\{F_{n}\right\}$ of finite rank operators in $C$ such that $\left\|F_{n}-K T\right\| \rightarrow 0$ as $n \rightarrow \infty$.

Let $S_{n}=A+F_{n} T^{-1}$. Then $S_{n}-T^{-1}=F_{n} T^{-1}-K$ and, given $\varepsilon>0$, there exists $N(\varepsilon)$ such that for $n>N(\varepsilon),\left\|S_{n}-T^{-1}\right\|<\varepsilon$. If $\varepsilon<1 /(|T|)$, it follows that $S_{n}$ is invertible and

$$
S_{n} T=A T+F_{n} T^{-1} T=A T+F_{n} \in C .
$$

Thus given $\varepsilon>0$, choose $B_{\varepsilon}^{-1}=S_{n}$ for $n>N(\varepsilon)$. This completes the proof.

EXAMPLE 1. Let $\not=l^{2}(0, \infty) \oplus l^{2}(0, \infty)$ with the nest whose projections are

$$
\left(\begin{array}{ll}
0 & 0 \\
0 & 0
\end{array}\right), \quad\left(\begin{array}{ll}
I & 0 \\
0 & 0
\end{array}\right), \quad\left(\begin{array}{ll}
I & 0 \\
0 & I
\end{array}\right) \text {. }
$$

Let

$$
T=\left(\begin{array}{cc}
U & e_{0} \otimes e_{0} \\
0 & U^{*}
\end{array}\right)
$$

where $e_{0} \otimes \epsilon_{0}$ is the projection on the one-dimensional subspace generated by $e_{0}$ and $U$ is the unilateral (forward) shift. Then $T$ is unitary and $T^{-1}$ is of the form $A+F$ with $A \in C \cap C^{*}$ and $F$ finite rank,

$$
T^{-1}=\left(\begin{array}{cc}
U^{*} & 0 \\
e_{0} \otimes e_{0} & U
\end{array}\right)
$$

Clearly, $T^{-1} \notin C$. 


\section{BIBLIOGRAPHY}

1. J. A. Erdös, Operators of finite rank in nest algebras, J. London Math. Soc. 43 (1968), 391-397.

2. T. Fall, W. Arveson and P. Muhly, Perturbations in nest algebras, J. Operator Theory 1 (1979), 137-150.

3. A. Feintuch and R. Saeks, Extended spaces and the resolution topology, Internat. J. Control 39 (1981), 347-354.

4. _ System theory - a Hilbert space approach, Academic Press, New York, 1982.

5. I. G. Gohberg and M. G. Krein, Theory and applications of Volterra operators in Hilbert space, Transl. Math. Monos., vol. 24, Amer. Math. Soc., Providence, R.I., 1970.

6. M. S. Lifshitz, On a certain class of linear operators in Hilbert space, Mat. Sb. 19 (1946), 239-262.

7. J. R. Ringrose, On some algebras of operators, Proc. London Math. Soc. 3 (1965), 61-83.

8. A. Feintuch, Invertibility in nest algebras, preprint.

Department of Mathematics, Ben Gurion University, Beersheva, Israel

Department of Mathematics, University of North Carolina, Charlotte, NORTH CAROLINA 28223 